\title{
A Generalization of Certain Properties of Laguerre Polynomials.
}

\author{
By B. Seetharama Sagtry.
}

(Received 17th September, 1943. Read 4th December, 1943.)

Let

$$
\Pi_{n}(z)=e^{z}\left(\frac{d}{d z}\right)^{n}\left\{e^{-z} A_{n}(z)\right\}
$$

where

$$
A_{n}(z)=a_{0} z^{n}+\left(\begin{array}{c}
n \\
1
\end{array}\right) a_{1} z^{n-1}+\left(\begin{array}{c}
n \\
2
\end{array}\right) a_{2} z^{n-2}+\ldots+a_{n},
$$

the $a$ 's being constants. Then the following relations hold:

(i) $\sum_{s=0}^{\nu}(-1)^{s}\left(\begin{array}{l}\nu \\ s\end{array}\right) \frac{n !}{(n-s) !} \Pi_{n-8}(z)$

and

$$
=(-1)^{n} \sum_{s=0}^{n-\nu}(-1)^{s}\left(\begin{array}{c}
n-v \\
s
\end{array}\right) \frac{n !}{(n-s) !} A_{n-s}(z),
$$

(ii) $\sum_{s=0}^{\nu}(-1)^{8}\left(\begin{array}{l}\nu \\ s\end{array}\right) \frac{n !}{(n-s) !} \Pi_{n-s}^{\prime}(z)$

$$
=-n \sum_{s=0}^{\nu-1}(-1)^{s}\left(\begin{array}{c}
\nu-1 \\
s
\end{array}\right) \frac{(n-1) !}{(n-s-1) !} \Pi_{n-s-1}(z),
$$

the accent denoting differentiation with respect to $z$. For, it follows from the definition of $\Pi_{n}(z)$ that

$$
\Pi_{n-s}(z)=\sum_{\nu=0}^{n-s}(-1)^{\nu}\left(\begin{array}{c}
n-s \\
v
\end{array}\right) \frac{(n-s) !}{\nu !} A_{\nu}(z) .
$$

Substituting this in the left-hand side of (i) and summing with respect to $s$, we obtain the right-hand side by Vandermonde's Theorem. Equation (ii) is obtained similarly on using the relation

$$
A_{\nu}^{\prime}(z)=\nu A_{\nu-1}(z) \text {. }
$$

It may be noted that (i) is of a reciprocal nature, being also valid when the $\Pi$ 's and $A$ 's are interchanged, as is evident on changing $\nu$ into $(n-\nu)$ on both sides.

Central College,

Bangalore, South India. 